\begin{tabular}{|c|c|c|c|c|c|}
\hline \multicolumn{6}{|c|}{ Sappho. } \\
\hline I 882 & & & U. $-\mathrm{L}$ & U. & L. \\
\hline Sept. 16 & + 106:34 & + 106". 1 3 & +0.21 & 8 & 2 \\
\hline Oct. I & +242.06 & +241.35 & +0.71 & 2 & 2 \\
\hline I & -55.29 & $-\quad 55.06$ & -0.23 & 2 & 4 \\
\hline 6 & +88.04 & +88.18 & -0.14 & 8 & 2 \\
\hline 7 & +309.86 & +309.15 & +0.71 & 8 & 2 \\
\hline
\end{tabular}

Wenngleich die Differenzen U. - L., als rein zufällige| unbeträchtlich und kann durch die Unsicherheit der ReFehler aufgefasst, einen etwas grösseren Werth des mittleren Fehlers ergeben, als aus den vorher abgeleiteten mittleren Fehlern erwartet werden konnte, so ist doch der Unterschied duction auf die Epoche Lund sowie auch durch die verschiedenartige Bestimmung des Schraubenwerthes erklärt werden.

Stockholm 1897 Mai.

Karl Bohlin.

\title{
Ephemeride des Winnecke'schen Cometen für die Erscheinung 1897-98.
}

\section{Von C. Hillebrand.}

Im Besitze der Manuscripte aus dem wissenschaftlichen Nachlasse Prof. v. Haerdtl's, die sich auf die Bearbeitung des periodischen Cometen Winnecke beziehen, bin ich in der Lage, für die nächste Erscheinung desselben eine genäherte Ephemeride zu geben, möchte aber einige Worte uber die Grundlagen derselben voranschicken.

Prof. v. Haerdtl hat für die Zeit von Anfang des Jahres 1892 bis Juni 1898 bereits die Jupitersstörungen gerechnet und zwar im unmittelbaren Anschluss an das Elementensystem, welches der Ephemeride für 1892 (A. N. Nr. 3 I I 2 ) zu Grunde liegt, so dass also die Resultate der Beobachtungen der letzten Erscheinung noch nicht berücksichtigt sind. Ich habe nun, um keine Verzögerung in der Veroffentlichung der Ephemeride eintreten zu lassen, unmittelbar das aus diesem Stadium der Rechnung resultirende Elementensystem benutzt und glaube, dass dasselbe als Grundlage einer Aufsuchungsephemeride völlig ausreichend sein wird. Es ist dies durch die geringe Abweichung der Beobachtungen von der Ephemeride 1892 und weiter durch den Umstand gerechtfertigt, dass während des letzten Umlaufes die Jupitersstörungen wohl die einzigen sind, die in einer genäherten Ephemeride merkbar sein dürften.

Was den ersten Umstand betrifft, so ergeben sich, wenn man aus den Beobachtungen von Algier, Berlin, München, Washington und Wien vor dem Perihel und aus denen von Windsor nach dem Perihel provisorische Normalörter bildet, folgende Abweichungen von der Ephemeride:

$$
\begin{array}{lcc}
\text { Vor dem } & \text { Perihel (1892 Juli } 1 \text { ). } \\
\text { I } 892 & \Delta \alpha \cos 8 & \Delta 8 \\
\text { April } 23.5 & -4.3 & -5.6 \\
\text { Mai } 24.5 & -3.0 & -4.0 \\
\text { Juni } 18.5 & -17.5 & -4.3 \\
\multicolumn{4}{c}{\text { Nach dem Perihel. }} \\
\text { Juli } 24.0 & -17.5 & +9.9 \\
\text { Aug. } 17.5 & -0.9 & +18.4 \\
\text { Sept. 23.5 } & +2.8 & +16.5
\end{array}
$$

Wenn man berücksichtigt, dass während der ganzen Dauet der hier benutzten Beobachtungen die Entfernung des
Cometen von der Erde kleiner als 0.6 des Erdbahnradius blieb und in der Erdnähe (Juli 9) sogar bis auf o.1 herabsank, so erscheinen diese Abweichungen als genügend klein, um die Weiterbenutzung des Elementensystems, wenigstens für den vorliegenden Zweck, zu rechtfertigen; mindestens kann aus einer provisorischen Rechnung keine reelle Verbesserung des Elementensystems erwartet werden.

In Betreff der Störungen, welche der Comet während des letzten Umlaufes erfahren hat, ist vor Allem die be. deutende Annäherung an Jupiter hervorzuheben. Der Comet stand nämlich Anfangs December 1894 in einem Abstand von diesem Planeten, welcher kleiner war als 0.5 des Erdbahnhalbmessers; ausserdem blieb aber die Entfernung durch mehr als vierzehn Monate (Mitte April 1894 bis Ende Juni 1 895) kleiner als 1.0. Die Störungen durch Jupiter sind dementsprechend bedeutende und erreichen für die Zeit 1892 Dec. I I bis 1898 Juni 3 folgende Beträge:

$$
\begin{aligned}
& \Delta M=+6^{\circ} \text { ' } 3^{\prime} 5^{\prime \prime} \text { ! I } \\
& \Delta \pi=-2 \quad 4 \quad 37.4 \\
& \Delta \delta=-3 \text { I8 } 24.6 \\
& \Delta i=+2284.6 \\
& \Delta \varphi=-0 \quad 5544.0 \\
& \Delta \mu=-1.235 \mathrm{I}
\end{aligned}
$$

Dem gegenüber stellt sich das Verhältniss $z u$ den übrigen Planeten, wie folgt:

Die Entfernung des Cometen von Saturn sinkt nicht unter 9.0, die Ende Februar 1898 stattindende Conjunction mit inbegriffen, und variirt zwischen diesem Werth und ca. 1 2.5. Dem Planeten Mars nähert sich der Comet erst bei der letzten Conjunction (April 1897) bis auf die Distanz 2.o. Was die Erde anbelangt, so kommt er derselben bei der letzten Conjunction (Ende Januar 1897) auf eine Entfernung von 3.0 nahe, hat noch Ende des Jahres 1897 einen Abstand von 1.8, welcher auch in der Erdnähe (Februar 1 898 ) nicht unter 1.3 sinkt. Uebrigens würde auch eine bedeutendere unberücksichtigt gelassene Störung um diese Zeit für die Auffindung von wenig Belang mehr sein. Von Venus und Mercur erreicht er im December 1897 die kleinste Distanz, die sich auf 2.8 , bezw. 2.3 beläuft. 
Die Ausgangselemente der Rechnung der Jupitersstörungen sind :

Epoche und Osculation I 892 Dec. I I.

$$
\begin{aligned}
& M=27^{\circ} 3^{6^{\prime}} 57^{\prime \prime} .8 \\
& \pi=276 \text { 10 } 56.6 \\
& \delta=104 \quad 3 \quad 32.9 \quad 1890.0 \\
& i=143130.8 \\
& \varphi=4633 \quad 4.0 \\
& \mu=609.7307
\end{aligned}
$$

Dabei ist zu bemerken, dass die Grössen $M$ und $\mu$ nicht ganz mit denjenigen übereinstimmen, die der Ephemeride für 1892 zu Grunde liegen, eine Differenz, die von einer verbesserten Mercursmasse herruhrt, mit welcher nachträglich die Störungen während des vorletzten Umlaufes gerechnet wurden.

Die nachstehende Ephemeride wurde nicht mit einem mittleren Elementensystem, sondern mit den jeweilig stattfindenden Elementen gerechnet, eine Genauigkeit, die allerdings in der hier gegebenen genäherten Form derselben belauglos sein dürfte.

Die beiden begrenzenden Elementensysteme sind:

$$
\begin{aligned}
1897 \text { Oct. } 26 \\
M=335^{\circ} 24^{\prime} 26.7 \\
\pi=274 \quad 1433.3 \\
\delta=10053 \quad 34.3 \\
i=165934.4 \\
\Phi=453735.6 \\
\mu=608.3483
\end{aligned}
$$

1898 März 15

$359^{\circ} 3^{\prime} 5^{2}$."

27414390

$100 \quad 53 \quad 11.5,1900.0$

$\begin{array}{lll}16 & 59 & 33.8\end{array}$

$45 \quad 37 \quad 14.1$

$608 . " 5559$
Die günstigste Position hat der Comet in der zweiten Hälfte December, wo er für unsere Breiten ungefähr $5^{\mathrm{h}} \cdot 5$ vor der Sonne aufgeht, allein erst Ende Februar erreicht er die Helligkeit, bei welcher er im Jahre 1892 aufgefunden wurde, um welche Zeit er nur mehr $3^{\text {h }}$ vor der Sonne aufgeht; dabei ist die Formel $I: \boldsymbol{r}^{2} \boldsymbol{\Delta}^{2}$ der Helligkeitsprognose zu Grunde gelegt. Günstiger gestaltet sich dieselbe, wenn man annimmt, dass die Entfernung von der Sonne die überwiegende Componente ist. In die der letzten Auffindung entsprechende Entfernung von der Sonne gelangt er nämlich bereits Anfangs December. Aller Wahrscheinlichkeit nach dürfte der Comet demnach in unseren Breiten wohl kaum ausser der Zeit Mitte December bis Mitte Februar gesehen werden können.

Ephemeride für $\mathbf{r}^{\mathrm{h}}$ M. Z. Berlin.

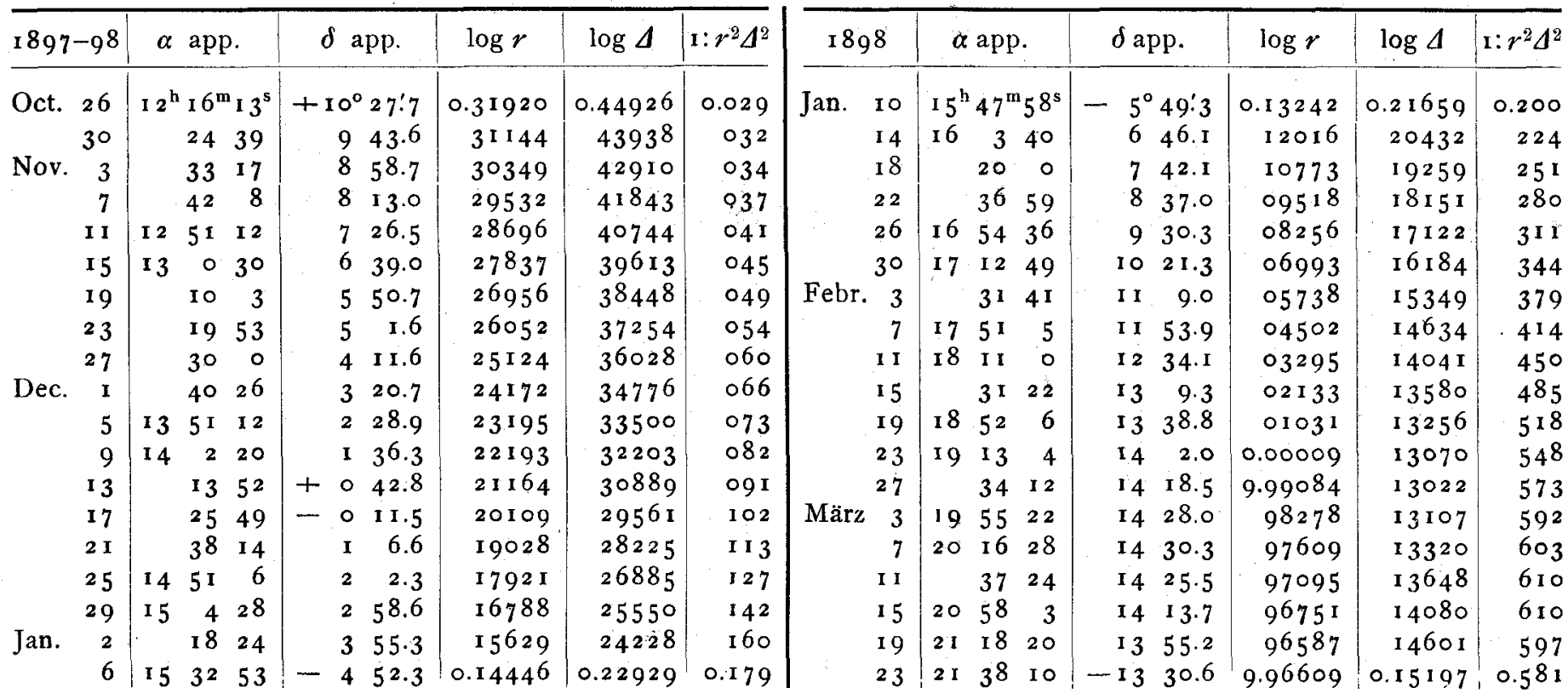

Wien, k. k. Sternwarte, 1897 August.

Perihel : 1898 März 20.6.

\section{Southern Double Stars.}

\section{By E.C. Pickering.}

A distinguishing feature of the climate of Arequipa is the great steadiness of the air. The value of this location as an observing station is largely due to this fact. Good definition, under high powers, is obtained there on many more nights than in Europe, or in the United States, where nine-tenths of the observatories of the world are at present located. A search for close double stars may, therefore, be advantageously made at Arequipa, and accordingly, in $189 \mathrm{r}$ all the stars of the sixth magnitude and brighter, south of declination $-30^{\circ}$, were examined for close companions. The stars in one quarter of the region, and included between $12^{\mathrm{h}}$ and $18^{\mathrm{h}}$ of right ascension were examined by Professor 